\title{
Recombineering strategies for developing next generation BAC transgenic tools for optogenetics and beyond
}

\author{
Jonathan T. Ting ${ }^{* \dagger}$ and Guoping Feng \\ McGovern Institute for Brain Research and Department of Brain and Cognitive Sciences, Massachusetts Institute of Technology, Cambridge, MA, USA
}

Edited by:

Mary Kay Lobo, University of

Maryland School of Medicine, USA

Reviewed by:

Michelle Gray, The University of

Alabama at Birmingham, USA

Joseph D. Dougherty, Washington

University School of Medicine, USA

\section{*Correspondence:}

Jonathan T. Ting, McGovern

Institute for Brain Research and

Department of Brain and Cognitive

Sciences, Massachusetts Institute

of Technology, 43 Vassar St.,

Cambridge, MA 02139, USA

e-mail: jtting@mit.edu

\section{†Present address:}

Jonathan T. Ting, Human Cell Types Program, Allen Institute for Brain

Science, Seattle, WA, USA
The development and application of diverse BAC transgenic rodent lines has enabled rapid progress for precise molecular targeting of genetically-defined cell types in the mammalian central nervous system. These transgenic tools have played a central role in the optogenetic revolution in neuroscience. Indeed, an overwhelming proportion of studies in this field have made use of BAC transgenic Cre driver lines to achieve targeted expression of optogenetic probes in the brain. In addition, several BAC transgenic mouse lines have been established for direct cell-type specific expression of Channelrhodopsin-2 (ChR2). While the benefits of these new tools largely outweigh any accompanying challenges, many available BAC transgenic lines may suffer from confounds due in part to increased gene dosage of one or more "extra" genes contained within the large BAC DNA sequences. Here we discuss this under-appreciated issue and propose strategies for developing the next generation of BAC transgenic lines that are devoid of extra genes. Furthermore, we provide evidence that these strategies are simple, reproducible, and do not disrupt the intended cell-type specific transgene expression patterns for several distinct BAC clones. These strategies may be widely implemented for improved BAC transgenesis across diverse disciplines.

Keywords: bacterial artificial chromosome, transgenic mice, BAC recombineering, Drd1a, Drd2, Adora2a, Chat, DAT

\section{INTRODUCTION}

Bacterial Artificial Chromosomes (BACs) are large DNA constructs composed of a small cloning vector backbone ligated to large fragments of restriction-digested genomic DNA that can be stably propagated as well as manipulated in bacterial host cells. Extensive BAC libraries have been constructed with genomic material from a variety of organisms and have served as indispensable tools for large-scale genome sequencing and mapping efforts. One such project culminated in the release of three landmark mouse BAC libraries derived from the C57BL/6J and 129S6/SvEvTac strains with a combined 30-fold coverage of the mouse genome (Osoegawa et al., 2000). This resource was quickly tapped to create the first $\mathrm{BAC}$ transgenic mouse line with functional transgene expression driven by successful integration of an engineered Bacterial Artificial Chromosome into the mouse genome (Yang et al., 1997). This pioneering work established the feasibility of diverse targeted manipulations of BAC clones in E. coli by homologous recombination, a method now commonly referred to as BAC recombineering (recombinationmediated genetic engineering). Furthermore, this work boldly asserted the incredible potential of BACs for gene therapy, disease modeling, and other basic research applications aimed at deciphering gene function.

The ability to obtain and manipulate BAC clones with genomic DNA spanning hundreds of kilobases is highly advantageous for transgenic applications given that most mouse BAC clones (average insert size 150-200 kb) encompass one or more genes including flanking regions essential to instructing cell-type specific expression patterns in vivo. Furthermore, the presence of large spans of insulating genetic material around a transgene expression cassette can curb transgene silencing or mosaic expression in vivo due to position effect variegation following random integration into unfavorable sites of the host genome (Bian and Belmont, 2010). Indeed, Yang et al. (1997) proposed that BAC transgenesis would be especially expedient for creating mice with faithful cell-type specific expression of Cre recombinase for gene disruption in mice, an idea that directly catalyzed the immensely successful Gene Expression and Nervous System ATlas (GENSAT) project at Rockefeller University led by Nathaniel Heintz and colleagues (Gong et al., 2003; Heintz, 2004). Importantly, BAC transgenic Cre driver rodents (particularly the extensive collection from the GENSAT project) have played a pivotal role in expanding the utility of emerging optogenetics-based technologies in neuroscience by enabling unprecedented access to monitor and manipulate genetically-defined cell populations in the nervous system when used in combination with Cre-inducible expression strategies for diverse optogenetic probes (Atasoy et al., 2008; Cardin et al., 2009; Petreanu et al., 2009; Witten et al., 2011; Madisen et al., 2012; Saunders et al., 2012; Zariwala et al., 2012). In addition, numerous laboratories have tapped into the knowledge base of the GENSAT project to guide the development of new tools such as the first collections of BAC transgenic mice with direct cell-type specific expression of ChR2 in the central nervous system (Hagglund et al., 2010; Zhao et al., 2011). Collectively, these BAC transgenic tools are now widely implemented for optogenetic deconstruction of complex neural circuits that mediate diverse animal behaviors and brain states (Tsai et al., 2009; Kravitz et al., 2010; Witten et al., 2010; Aponte et al., 2011; Halassa et al., 
2011; Yizhar et al., 2011; Tai et al., 2012; Tan et al., 2012; Bock et al., 2013; Chaudhury et al., 2013; Cui et al., 2013; Stamatakis et al., 2013; Steinberg et al., 2013). The broader topic of optogenetic dissection of behavior in mammalian model systems has been extensively reviewed elsewhere (Tye and Deisseroth, 2012; Yizhar, 2012; Lenz and Lobo, 2013; Nieh et al., 2013).

Here we propose strategies for developing the next generation of BAC transgenic lines that are devoid of overexpressed extra genes. We describe multiple BAC recombineering strategies for eliminating undesirable extra genes from BAC clones in order to circumvent confounds due to overexpression of such extra genes in BAC transgenic lines. In addition, we demonstrate that these modification procedures may be performed in parallel or sequentially with routine $\mathrm{BAC}$ recombineering steps for introducing a transgene of interest under the control of cell-type specific promoter elements. Together, these procedures effectively result in pure transgenic expression cassettes $\geq 65 \mathrm{~kb}$ in size that can be used in pronuclear injection to produce BAC transgenic animals. These steps are simple, efficient, reproducible, and can be implemented for the modification of any BAC clone. We are applying these methods in developing the next generation of BAC transgenic animals for optogenetics-based research and expect these strategies may be widely adapted across diverse disciplines.

\section{MATERIALS AND METHODS TARGETING VECTOR CONSTRUCTION}

A pBlueScript-derived vector iTV1 was used to generate BAC targeting vectors. iTV1 contains cloning sites for A and B homology arms which flank a large multiple cloning site, bovine growth hormone polyadenylation (BGHpA) signal, and FRT flanked neomycin resistance cassette (FRT-NEO-FRT). First, iTV1 was modified by addition of the woodchuck post-translational regulatory element (WPRE) upstream of the BGHpA to create iTV1-WPRE. This modification was chosen to improve transgene protein expression level and RNA stability. BAC specific homology arms A and B (400-600 bp each arm) were PCR amplified from BAC template DNA and cloned into iTV1WPRE. In a separate mammalian expression vector, two copies of ChR2(E123T/H134R), herein referred to simply as ChETA TR $_{\text {, and }}$ the orange/red emitting tdTomato fluorophore were combined using viral 2A linkers. We selected P2A with a C-terminal GSG linker because this combination was shown to be the most efficiently processed of all viral 2A elements (Kim et al., 2011) and thus was the most likely to enable a complete physical uncoupling of the opsin and fluorophore with no significant unprocessed protein fraction. The $\mathrm{ChETA}_{\mathrm{TR}}-\mathrm{P} 2 \mathrm{~A}-\mathrm{ChETA}_{\mathrm{TR}}-\mathrm{P} 2 \mathrm{~A}$-tdTomato cassette (i.e., $2 \mathrm{xChETA-P2A-tdTomato)} \mathrm{was} \mathrm{then} \mathrm{sub-cloned} \mathrm{into} \mathrm{the}$ large multiple cloning site of iTV1-A/B-WPRE to complete the BAC targeting vectors. In this final cloning step great care was taken to ensure minimal disruptions to the junction between the A Box and the start of the ChETA sequence, except for addition of a Kozak consensus sequence.

\section{BAC TRIMMING CASSETTES}

The pBlueScript-based deletion cassettes were constructed by standard PCR and cloning methods. The order of assembled features is as follows: (1) a 50-350 bp BAC-specific homology arm (selected to target the preferred location for BAC trimming in a BAC clone of interest), (2) AscI and NotI restriction sites (for later removal of the BAC vector during purification of BAC DNA for pronuclear injection), (3) the bla gene encoding ampicillin resistance (AmpR), and (4) a "loxP deletion" homology arm (targeting the BAC vector sequence adjacent to the wild-type loxP site in pBACe 3.6 or pTARBAC1 vectors).

loxP deletion arm: 5 '-gttaacgtgccggcacggcctgggtaaccaggtatttgtc cacataaccgtgcgcaaaatgttgtggataagcaggacacagcagcaatccacagcaggcat acaaccgcacaccgaggttactccgttctacaggttacgacgacatgtcaatacttgccettgac aggcattgatggaatcgtagtctcacgctgatagtctgatcgacaatacaagtg- $3^{\prime}$

BAC clone specific deletion arms:

Adora2a BAC (RP24-238K3): 5' -gagctgagtggccagcgacctattgcc taggcatagataaccatatatca- $3^{\prime}$

Drd2 BAC (RP23-161H15): 5'-gagaccagtgccagcagaagctatggtc attgtggtgataggagcgtggctga- $3^{\prime}$

Chat BAC (RP24-256F2): 5'-ttcagtccactatactttcctgctttcttcatg gcttagcaaggttcctggacctcagcagagttaatagaaaatgcaggctgcaactggatggtt agcgatgaaactaagcaactctagacagtgcagtcagacacatactttctctaactggcggga gttactcactcacgcaatcacctctaacacttaaccacagcaggtggaaattgagttagtttaa gaggctaactctgtgctaagcctggggacttgggacaggaaagccttggccccgcccagcag tggcccocccacctctctgaaggctggactggcggttgcctagcagcagg- $3^{\prime}$

DAT BAC (RP24-269I17): 5' -agatataacctacctttgcatgttagccagga ttaagatttatattaccg- $3^{\prime}$

Pvalb BAC (RP24-306A6): 5'-ttccggaaggtgcacagcacgggtgctgtc cgcagttgtttgctgtgcaagcatgcagggccttcctgtcttcctcactgctcaacgttgcacat ttttccctcccegctatttcagtttttagggttcataacatgcttgggctttaaagatggcatttcg attctggacgtgaa- $3^{\prime}$

Vgat BAC (RP24-246L1): 5'-agttccatccctggaacctattgggtgaagag aacaggcatcttggcacgctcccacacccatcacgcaataaataaacaaatgaacagaaaaa gaagtcaaaggggaaggagattagcttaacacggtcacaaaactaagggctagatctgcaag gctatgcagacagagaccaagggagagtaaggggacagggaggggcggagtccagcccag tgtgggtggagccttggtcccttagttagagca- $3^{\prime}$

\section{GENE INACTIVATION CASSETTES}

Sfxn1 homology arms A and B (1 kb each) were PCR amplified and cloned into pBlueScript II. A $29 \mathrm{bp}$ dsOligo containing a three-frame translational stop mutation and diagnostic SalI restriction site ( $5^{\prime}$-gaattctagataactaggtcgacctgcag- $\left.3^{\prime}\right)$, was synthesized and cloned between the two homology arms. This vector is designed to insert the three-frame translational stop cassette after the 13th codon in the Sfxn 1 coding region in the Drdla-spanning BAC clone RP23-47M2 and was thus called "Sfxn1 Target." A second vector "VAChT Target" was designed with the identical strategy except that homology arms A and B directed the insertion of the three-frame translational stop cassette after the 5th codon in the Slc18a3 coding region (VAChT locus) in the Chatspanning BAC clone RP24-256F2. Relatively long $1 \mathrm{~kb}$ homology arms were selected in order to ensure a high efficiency of homologous recombination since this targeting cassette does not contain a selection marker for screening and must be used in combination with a separate iTV1-based targeting vector.

Critical regions of all constructs were verified by DNA sequencing. The targeting vectors were linearized by restriction digestion and purified in preparation for subsequent use in BAC recombineering steps. Care was taken to ensure adequate duration of restriction digestion (or in some cases two sequential 
rounds of digestions were performed) such that there was no contaminating uncut plasmid carried over into the electroporation step.

Additional information including complete DNA sequences of the targeting vectors and modified BAC clones used in this study are available upon request.

\section{BAC RECOMBINEERING}

Appropriate BAC clones were selected from the RP23/RP24 C57 mouse BAC library following a detailed analysis using the Esembl Genome Browser (www.Ensembl.org) and were obtained from the Children's Hospital Oakland Research Institute (CHORI). The intact BAC DNA was isolated from the original host DH10B strain by BAC mini-prep and verified by restriction digestion and pulsed field gel electrophoresis (PFGE). Successfully verified BAC DNA was transferred into the EL250 strain in preparation for recombineering steps and maintained as glycerol stocks stored at $-80^{\circ} \mathrm{C}$.

Most often the first strategic recombineering step was BAC trimming to delete unwanted regions of BAC DNA harboring extra genes. $20 \mathrm{~mL}$ of LB media plus chloramphenicol were inoculated with EL250 cells containing the target BAC clone and placed in a shaking incubator at $32^{\circ} \mathrm{C}$ for $2 \mathrm{~h}$ until the cells reached an early log growth phase. The culture was induced for $15 \mathrm{~min}$ at $42^{\circ} \mathrm{C}$ in a shaking water bath to permit transient expression of genes required for homologous recombination. Cells were rapidly chilled, pelleted, and washed three times with ice-cold MilliQ water to remove salts. Approximately 10-100 ng of linearized BAC trimming vector was added to $50 \mu \mathrm{L}$ of induced cells in a $1 \mathrm{~mm}$ gap cuvette and the mixture was immediately electroporated $(1.75 \mathrm{kV}, 25 \mu \mathrm{F}, 200 \mathrm{Ohms})$. The cells were recovered and plated onto LB plus chloramphenicol and carbenicillin to select for successful homologous recombination events, meaning insertion of the AmpR cassette in place of the targeted region. Double resistant clones were picked for further analysis to verify the deletions.

In a second round of targeting the virtually identical steps were performed to insert the $C h E T A_{\mathrm{TR}}$ transgene expression cassette at the initiating methionine start codon of the targeted gene within the selected BAC clone by homologous recombination. In this case, successfully modified clones were resistant to chloramphenicol, carbenicillin, and kanamycin due to insertion of the FRT-NEO-FRT in the targeting vector. (Note: although it is easily feasible to perform the first and second targeting events in a single step due to the insertion of two different selection cassettes, it was preferable to do these steps in series in order to save a glycerol stock of the successfully trimmed BAC in EL250 cells for future reuse). The NEO cassette was then excised from the modified BAC by arabinose induction of flp recombinase in the EL250 cells. Modified BAC clones were extensively screened for accuracy and the correctly targeted BAC DNA was grown in large scale and purified using the BAC100 kit (Clontech). 10-15 $\mu \mathrm{g}$ of BAC DNA was restriction digested with NotI or AscI to liberate the $\mathrm{BAC}$ vector from insert and the linear fragments were separated by PFGE. The intact BAC insert band was excised from the PFG, electroeluted, and spot dialyzed against fresh microinjection buffer.
For gene inactivation recombineering experiments the linearized Sfxn1 Target DNA was combined at $\sim 20$-fold molar excess with linearized iTV1-D1-ChETA targeting construct DNA immediately prior to the electroporation step. This was best accomplished by diluting linearized iTV1-D1-ChETA targeting DNA 1:20 in water and then adding approximately equal volumes of linearized Sfxn1 Target and diluted iTV1-D1-ChETA into the cuvette for the electroporation into induced EL250 cells propagating the Drd1a-spanning BAC RP23-47M2. Double recombinants were identified by resistance to chloramphenicol and kanamycin and further verified by PCR screening and subsequent restriction digestion and PFGE of isolated BAC DNA to test for the incorporation of the diagnostic SalI site. Large scale BAC DNA isolation and processing for pronuclear injection were carried out as indicated above. In additional experiments we used VAChT Target to inactivate Slc18a3 in the Chat-spanning BAC RP24-256F2. This dual targeting step was carried out together with the iTV1-ChatChETA targeting vector following identical methods as for Sfxn1 inactivation.

\section{PRONUCLEAR INJECTION AND IDENTIFICATION OF TRANSGENIC FOUNDERS}

Transgenic mice (pure C57BL/6 Taconic) were generated by pronuclear injection of the highly purified intact BAC DNA into fertilized oocytes at a concentration of $0.5-2.0 \mathrm{ng} / \mu \mathrm{L}$. Genotypes were determined by PCR from mouse tail DNA samples and linespecific primer sets. Mice that had the transgene integrated in the genome were kept as founders to establish distinct lines by mating to C57BL/6J mice. One line each of Slc6a3-2xChETA ${ }_{T R^{-}}$ P2A-tdTomato (DAT-ChETA), Drd1a-2xChETA ${ }_{T R}-P 2 A$-tdTomato

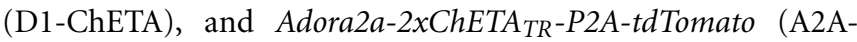
ChETA) were established for further analysis. Only hemizygous mice on a pure C57 background were used for experiments in this study. All research involving mice was conducted according to the Institutional Animal Care and Use Committee guidelines at MIT. All procedures were approved by the Institutional Animal Care and Use Committee at MIT.

\section{SLICE IMMUNOSTAINING AND CONFOCAL IMAGING}

Mice were anesthetized with tribromoethanol (Avertin) and transcardially perfused with phosphate buffered saline $(137 \mathrm{mM}$ $\mathrm{NaCl}, 10 \mathrm{mM} \mathrm{NaH} \mathrm{PO}_{4}$, and $2.7 \mathrm{mM} \mathrm{KCl}, \mathrm{pH}=7.4 ; \mathrm{PBS}$ ) followed by $10 \%$ neutral-buffered formalin (Sigma-Aldrich, St Louis, MO). Brains were post-fixed in $10 \%$ formalin at $4^{\circ} \mathrm{C}$ overnight. Fixed brains were sectioned $(50 \mu \mathrm{m})$ on a vibratome (Leica Microsystems, Buffalo Grove, IL) and the free-floating sections were washed for $10 \mathrm{~min}$ three times with PBS. Sections were then blocked for $1 \mathrm{~h}$ at room temperature in PBS containing 5\% normal goat serum, $2 \%$ bovine serum albumin and $0.2 \%$ Triton-X 100. After blocking, the sections were incubated overnight at $4^{\circ} \mathrm{C}$ in blocking buffer containing one or more of the following antibodies: anti-ChAT (1:200 dilution; Millipore AB144P), anti-2A (1:500 dilution; Millipore ABS31), anti-red fluorescent protein (1:500 dilution; Rockland 600-401-379), antiGFP (1:5000 dilution; Abcam AB6566), anti-DAT (1:300 dilution; Millipore MAB369), or anti-DARPP-32 (1:2000 dilution; BD Transduction 611520). Following the overnight incubation, 
sections were washed for 20 min three times with PBS and then incubated for $1 \mathrm{~h}$ at room temperature in Alexa dye-conjugated secondary antibody (1:1500; Invitrogen, Grand Island, NY). Alexa dyes utilized were: Alexa Fluor 488 (A-10667, goat anti-mouse and A-11034, goat anti-rabbit), and Alexa Fluor 555 (A-21429, goat anti-rabbit). Sections were washed for $20 \mathrm{~min}$ three times with PBS, dried on a glass microscope slide and mounted with VectaShield (Vector Laboratories, Burlingame, CA). Slides were stored at $-20^{\circ} \mathrm{C}$ and were thawed to room temperature immediately prior to imaging. Whole brain montage images were acquired with CellSens software on an Olympus BX61 equipped with a motorized stage and epifluorescence illumination. Confocal z-stack images were acquired on an Olympus Fluoview 1000 laser scanning confocal microscope equipped with 488 and $543 \mathrm{~nm}$ laser lines.

\section{RESULTS}

\section{IMPLEMENTING WELL CHARACTERIZED BAC CLONES TO ACHIEVE REPRODUCIBLE CELL-TYPE SPECIFIC TRANSGENE EXPRESSION}

We have previously developed several BAC transgenic mouse lines for expression of $\mathrm{ChR} 2$ in diverse neuronal subsets of the nervous system (Zhao et al., 2011). Our general strategy has been to select BAC clones that have previously been validated in the GENSAT project, as determined by the successful development of mouse lines for cell type-specific expression of Cre or EGFP. With this strategy we have observed that independent BAC transgenic mouse lines created by modification of the same original BAC clone exhibit highly reproducible cell-type specific transgene expression that recapitulates endogenous gene expression patterns. This is exemplified in the case of the Chat-spanning BAC clone RP23-246B12, which was successfully used to express both EGFP and ChR2-EYFP in cholinergic neurons of the nervous system (Figure 1). Thus, a known BAC clone can be modified for expression of virtually any transgene of interest in a welldefined neuronal subset with a relatively high probability of success following pronuclear injection.

\section{MOST BAC CLONES USED TO MAKE BAC TRANSGENIC LINES SPAN MULTIPLE GENES}

In the course of this work it was noted that the majority of BAC clones selected for making BAC transgenic mice and rats contained multiple extra genes in addition to the explicitly targeted region (Table 1). Presumably this relates to the conventional wisdom that larger BAC clones spanning the targeted region are more likely to encompass the necessary regulatory elements for recapitulating the desired transgene expression pattern. Thus, in order to more broadly assess this issue of increased gene dosage for off-target genes we analyzed the entire collection of BAC clones from the GENSAT Cre driver mouse repository (http://www. gensat.org/cre.jsp). In total, $139 \mathrm{BAC}$ clones from the RP23/RP24 C57 library were located and viewed with alignment to the mouse genome using the Ensembl Genome Browser (http://www. ensembl.org). This enabled us to determine the number of extra genes (i.e., genes other than the targeted gene region) spanned by each unique BAC clone. We report that $75 \%$ of the BAC clones used to derive GENSAT Cre driver lines contain at least one or more extra gene (Figure $\mathbf{2 A}$ ), and in the extreme cases



up to 14 independent and complete extra genes were spanned by a single BAC clone (e.g., RP23-368D24 used to create ThbsCre lines SW48-Cre and SW52-Cre; Figure 2B). Only 25\% of BAC clones were entirely devoid of extra genes $(12 \%)$ or only contained incomplete portions of gene coding regions (13\%). Notably, many of the analyzed BAC clones used to derive GENSAT Cre driver lines were also previously implemented for making EGFP reporter lines.

\section{EXTRA GENES CONTAINED WITHIN BAC CLONES CAN RESULT IN UNINTENDED GENE OVEREXPRESSION IN BAC TRANSGENIC LINES}

The infrequently discussed down side of selecting very large BAC clones for BAC transgenesis is the potential for overexpression of unwanted extra genes and any associated behavioral, biochemical, or electrophysiological confounds. We previously 
Table 1 | BAC transgenic lines typically have increased gene dosage of one or more extra gene.

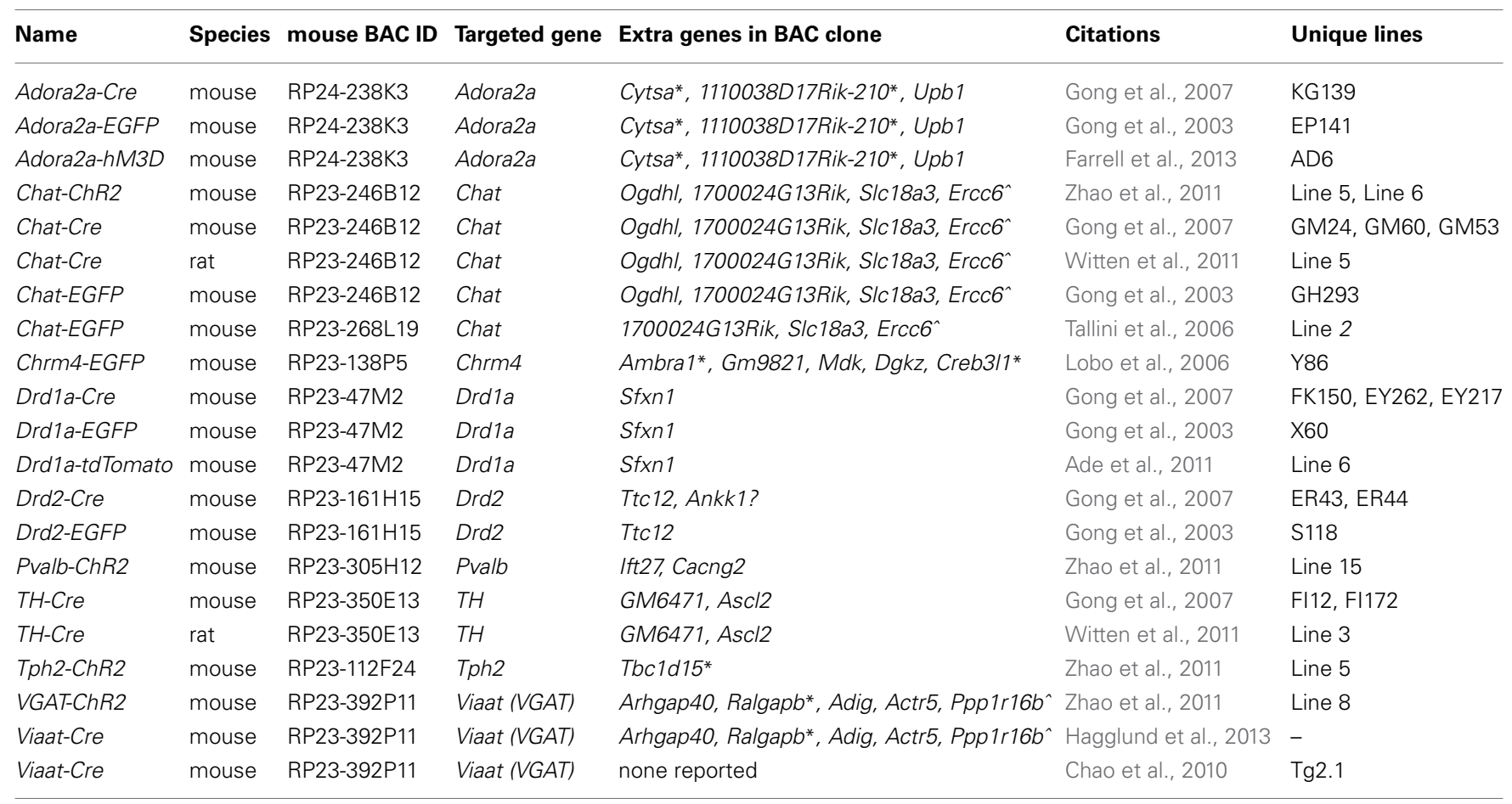

${ }^{*}$ Partial gene that is missing the first coding exon containing ATG.

"Truncated gene.

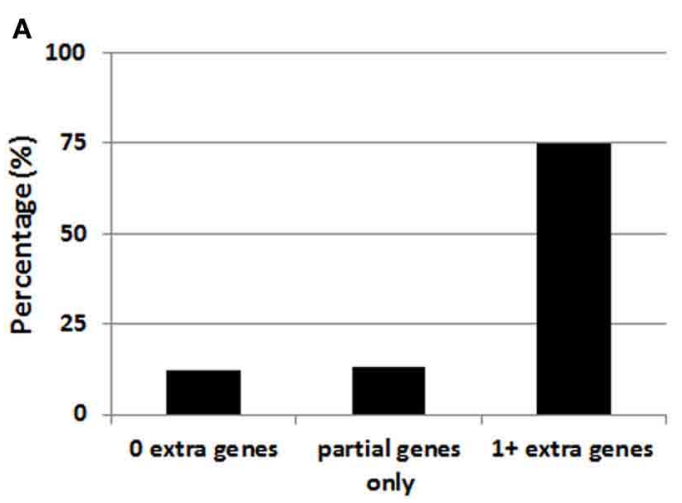

FIGURE 2 | Analysis of extra genes spanned by BAC clones of the GENSAT Cre driver repository. (A) Plot of the percentage of BAC clones with either zero, partial gene fragments only, or 1 or more extra genes

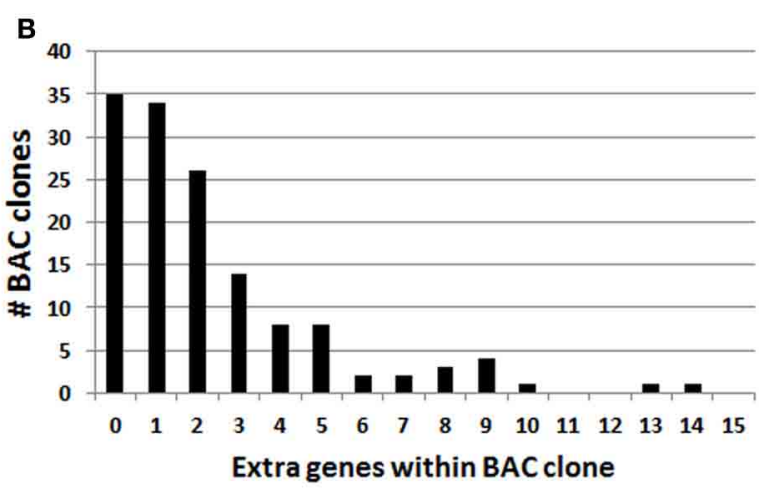

spanned. (B) Plot of the full distribution of extra gene number for the 139 BAC clones analyzed. Only intact genes that were fully contained within the BAC clone were counted. developed a ChAT-ChR2-EYFP BAC transgenic mouse line (line 6) that enables optogenetic control of cholinergic neuron firing in vitro and in vivo (Ren et al., 2011; Zhao et al., 2011; Ma and Luo, 2012). The BAC clone that was used to create our ChR2-EYFP line was the same as the clone used to create independent ChAT-Cre mouse and rat lines (Gong et al., 2007; Witten et al., 2011). This Chat-spanning BAC clone contains the additional genes Ercc6 (partial), Ogdhl, 1700024G13Rik and Slc18a3 (Figures 3A-C). Importantly, the Slc18a3 gene is nested within the first intron of the Chat gene and encodes for the vesicular acetylcholine transporter (VAChT), the overexpression of which has profound implications for cholinergic function. This prediction was confirmed in a recent study that provided direct evidence for increased VAChT expression in ChAT-ChR2-EYFP line 6 mice, and linked this VAChT overexpression to increased cholinergic tone, enhanced motor endurance, and attention and memory deficits (Kolisnyk et al., 2013). More specifically, the authors reported a 20-fold increase in VAChT transcript level in striatum and a 5-fold increase in VAChT protein level in hippocampus with no significant elevation of ChAT expression in 


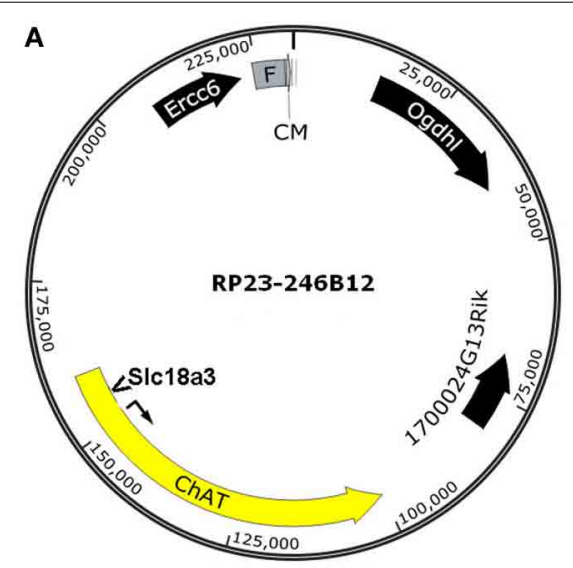

c

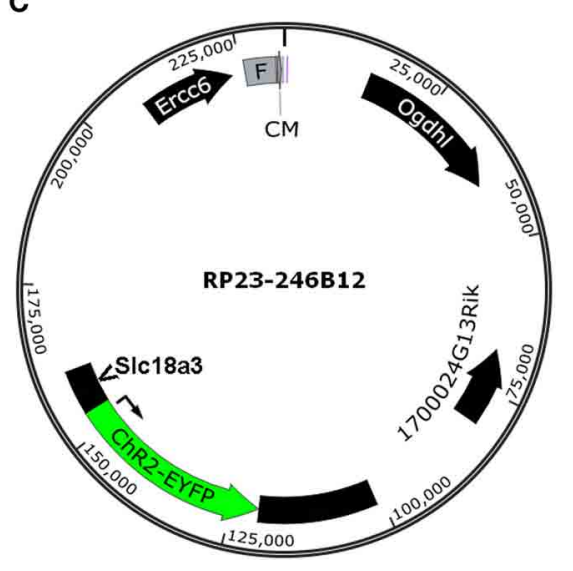

FIGURE 3 | Extra genes contained within BAC clones can result in unintended gene overexpression in BAC transgenic lines. Simplified diagrams of the Chat-spanning BAC clone RP23-246B12 (A) that was modified for expression of (B) Cre recombinase (GENSAT BAC ID $\mathrm{BX} 1191)$ or (C) ChR2(H134R)-EYFP under the control of the Chat gene promoter elements. Several extra genes are contained in the BAC clone, including Ercc6 (partial), Ogdhl, 1700024G13Rik, and Slc18a3. Notably,

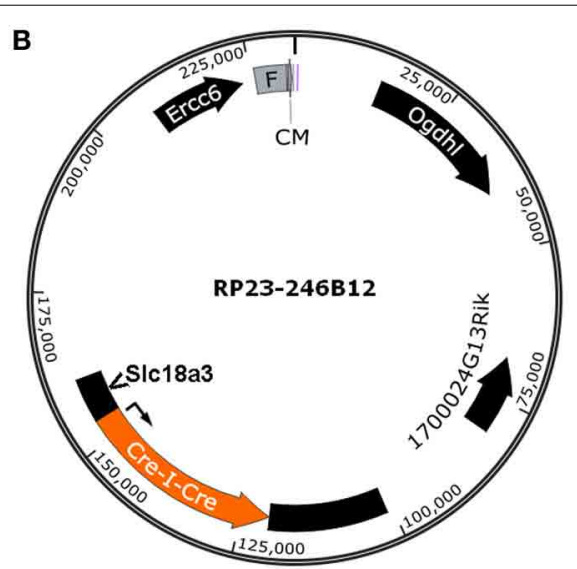

D

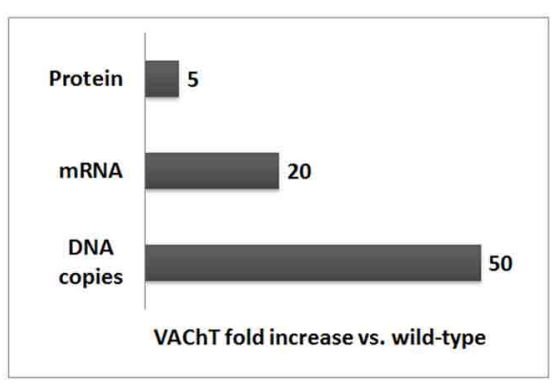

Slc18a3 (the gene encoding VAChT) is nested within the first intron of the Chat gene. (D) Summary of fold changes in VAChT levels (DNA copies, mRNA, and protein) in brain tissue from Chat-ChR2(H134R)-EYFP line 6 BAC transgenic mice relative to non-transgenic controls. This chart is based on data presented in Kolisnyk et al. (2013).

Abbreviations: F, F-factor replicon; CM, chloramphenicol resistance gene. Both elements are part of the BAC vector. either region (summarized in Figure 3D). The protein overexpression was associated with a 3 -fold increase in stimulus-evoked ACh release in hippocampal slices, consistent with the behavioral data that VAChT overexpression has important functional consequences (Kolisnyk et al., 2013). Furthermore, striatal VAChT overexpression and elevated $\mathrm{ACh}$ release are associated with increased severity of psychomotor stimulant-induced repetitive behaviors in ChAT-ChR2-EYFP line 6 BAC transgenic mice (Jill R. Crittenden and Ann Graybiel, pers. commun.).

\section{STRATEGY FOR DELETION OF EXTRA GENES BY BAC TRIMMING}

The most straightforward approach for eliminating extra genes contained in BAC clones is to remove segments from either end of the BAC insert, a method known as BAC trimming or shaving. We implemented a versatile method for BAC trimming in which small antibiotic selection cassettes are used to replace precise BAC DNA segments using a recombineering step in E. coli (Hill et al., 2000; Testa et al., 2003, 2004). The selection cassettes were PCR amplified using long primers to add $\sim 50$ bp homology arms to either end, and then used for recombineering in EL250 cells. The homology arms define the exact BAC region that will be deleted by the insertion of the selection cassette, and the selection cassette enables subsequent identification of properly modified BAC clones. In addition, many different selection cassettes can be utilized for BAC trimming (e.g., ampicillin resistance-bla, kanamycin resistance-neo, chloramphenicol resistance-cam, blasticidin resistance- $b s d$, and streptomycin resistance- $r p s L$ ), providing options for sequential rounds of BAC trimming and transgene targeting using different selection markers. To further generalize this strategy for deleting fragments from any BAC clone in the RP23 and RP24 library, in subsequent experiments one of the homology arms was designed on the end of the BAC vector such that only the second homology arm needs to be customized for each unique BAC clone to define the deletion region-the region containing undesirable extra genes. In addition, we added NotI and AscI restriction sites adjacent to the unique homology arm but before the selection cassette. Once the deletion of the BAC segments was confirmed by restriction digestion and pulsed field 
gel electrophoresis (PFGE) the BAC insert was liberated from the BAC vector and selection cassette by NotI or AscI digestion and subjected to further purification. We have used this method to successfully delete precisely defined BAC DNA segments, as demonstrated for the Slc6a3-spanning BAC clone RP24-269I17 in which the extra genes Clptm1l, Tert, Slc6a18, and Slc6a19 located in the $3^{\prime}$ flanking region of the Slc6a3 (DAT) gene were deleted by homologous recombination in which an $\sim 84 \mathrm{~kb}$ BAC region was replaced with a $1 \mathrm{~kb}$ ampicillin resistance $(\mathrm{AmpR})$ cassette (Figures 4A-C). The "trimmed" BAC clone can then be used for insertion of a transgene cassette in place of the Slc6a3 gene by standard BAC recombineering using an iTV-based targeting vector (see Materials and Methods). The successful DAT BAC trimming was verified by diagnostic restriction digestion with SalI and PFGE (Figure 4D).

It is worth noting that other methods have been described for removal of extra genes from $\mathrm{BAC}$ clones to produce $\mathrm{BAC}$ transgenic mouse lines. In one study a modified BAC clone was subjected to restriction digestion and PFGE, and fortuitously, a fragment could be excised that contained suitably large $5^{\prime}$ and $3^{\prime}$ flanking regions but excluded several extra genes originally present in the BAC clone (Chao et al., 2010). In another study a spontaneous deletion was identified in a single clone during the recombineering steps, and this deletion serendipitously removed the only extra gene present in the BAC clone (Belforte et al., 2010). However, the recombineering-based method we have outlined for
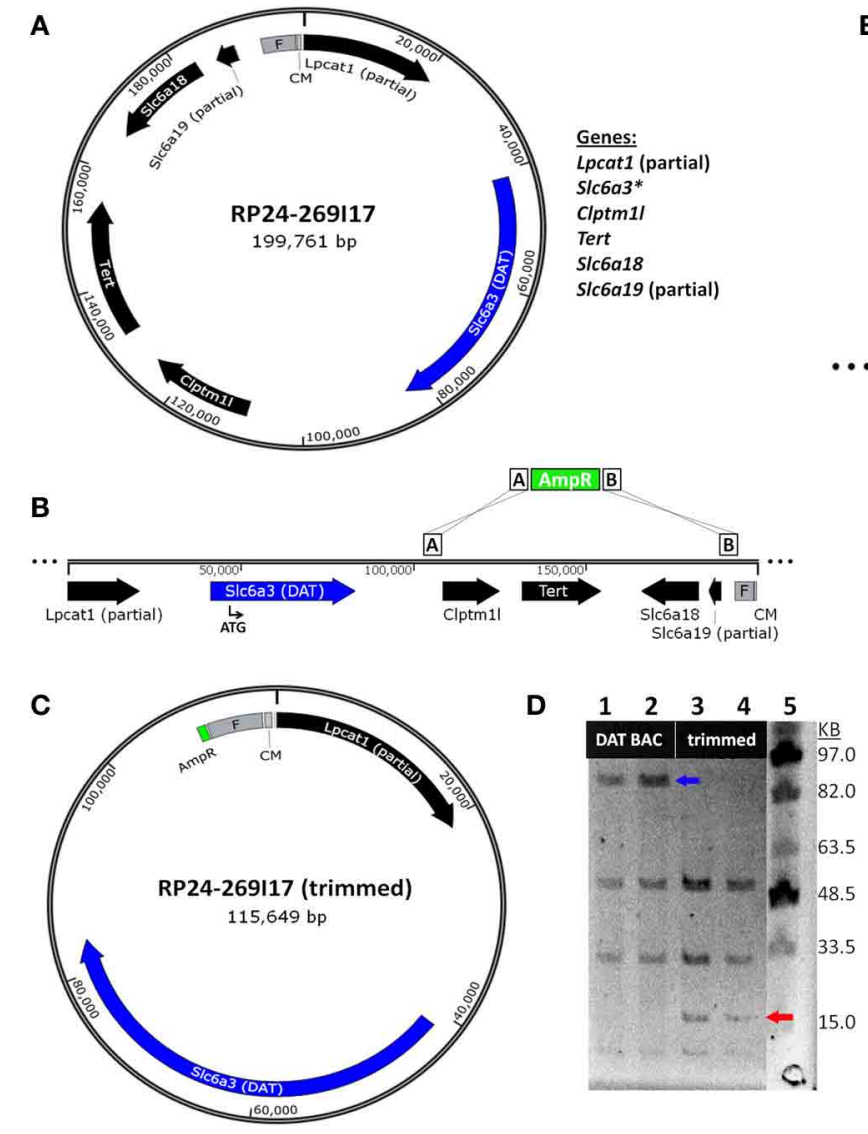

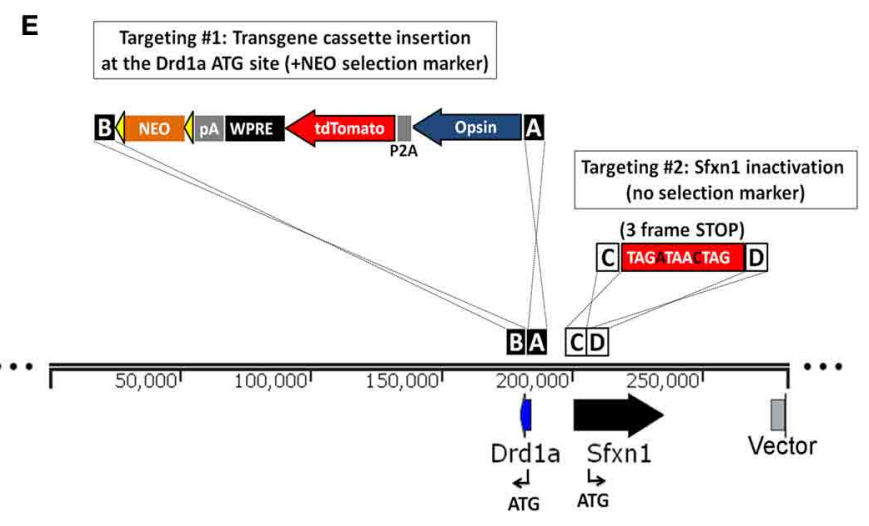

F

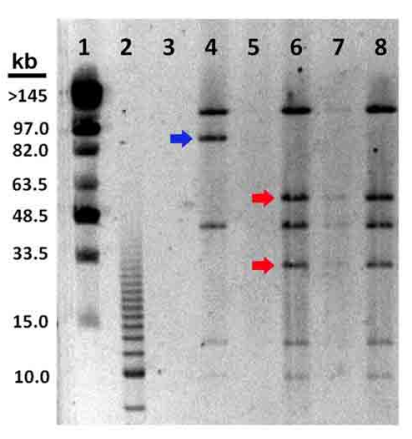

FIGURE 4 | BAC recombineering strategies for avoiding expression of extra genes. (A) Strategy for deletion of extra genes by BAC trimming. A diagram of the DAT (SIc6a3)-spanning BAC clone RP24-269117 reveals the presence of many extra genes including Clptm1I, Tert, Slc6a18 and the truncated genes Lpcat1 and S/c6a3. (B) The extra genes Clptm11, Tert, S/c6a18, and S/c6a19 located in the 3' flanking region of the DAT gene are deleted by homologous recombination. The recombination event replaces the $\sim 84 \mathrm{~kb}$ BAC region with a $1 \mathrm{~kb}$ ampicillin resistance (AmpR) cassette. (C) Diagram of the "trimmed" DAT BAC clone that can now be used for insertion of a transgene cassette in place of the DAT gene by BAC recombineering. (D) Verification of DAT BAC trimming by diagnostic restriction digestion with Sall and PFGE. Lane 1 and 2: modified DAT BAC DNA. Lane 3 and 4: trimmed DAT BAC DNA. Lane 5: MidRange I PFG Marker. In successfully trimmed BAC clones the $91 \mathrm{~kb}$ DNA fragment (blue arrow) is reduced to $17 \mathrm{~kb}$ (red arrow). In addition, a 6 and $4 \mathrm{~kb}$ fragment are deleted but are too small to be visualized on the PFG. (E) Strategy for inactivation of extra genes by dual targeting. The Drd1a-spanning BAC clone RP23-47M2 contains the extra gene $S f x n 1$ in the promoter region of the Drd1a gene. In the dual targeting strategy, two simultaneous homologous recombination events must occur. The first targeting event is the insertion of the transgene cassette (including the NEO selection marker) in place of the Drd1a gene. The second targeting event is the insertion of a triple frame stop mutation (and Sall restriction site) immediately downstream of the Sfxn1 start codon. Double recombinant clones are identified by kanamycin resistance. (F) Verification of Sfxn1 gene inactivation in modified Drd1a BAC DNA by diagnostic restriction digestion with Sall and PFGE. Lane 1: MidRange I PFG Marker. Lane 2: $2.5 \mathrm{~kb}$ marker. Lane 4: Drd1a BAC DNA (+Sfxn1). Lanes 6-8: Drd1a BAC DNA following $S f x n 1$ inactivation. For successfully modified BAC clones the $\sim 95 \mathrm{~kb}$ DNA fragment (blue arrow) is cleaved into 60 and $35 \mathrm{~kb}$ fragments (red arrows). Note that all other bands remain unaltered. 
BAC trimming affords greater reliability and precision for routine deletion of extra genes.

\section{INACTIVATION OF POTENTIALLY CONFOUNDING "EXTRA" GENES BY MINIMAL INSERTION MUTATIONS}

Although BAC trimming can be a versatile and powerful method for deleting both small and large segments of BAC DNA, in some cases it is not permissible to apply this method for the removal of extra genes. For example, when an extra gene is located within the critical $5^{\prime}$ and $3^{\prime}$ flanking regions of the targeted gene, deletion of these flanking regions would likely negatively affect the pattern of gene expression. Thus, we devised a recombineering strategy to inactivate the $S f x n 1$ gene nested within the putative promoter region of the Drd1a gene by inserting stop codons in all three reading frames to interrupt the coding region of $S f x n 1$. In this manner the inactivation of an extra gene can be carried out with minimal insertions as small as $\sim 15 \mathrm{bp}$, but in our case we have designed a 29 bp sequence (see Materials and Methods) for targeted inactivation of $S f x n 1$ gene in the Drd1a-spanning BAC RP24-47M2 that further introduces unique restriction sites for diagnostic purposes (Figure 4E). The targeting vector for gene inactivation does not contain any selection marker and therefore must be used in conjunction with a second targeting vector that does contain a selection marker, in this case either an iTV-based targeting vector or a BAC trimming cassette.

In order to ensure that the antibiotic selection appropriately isolates double recombinant clones, the concentrations of the linearized targeting vectors were carefully proportioned. The linearized Sfxn1 Target and iTV1-D1-ChETA Target DNA were combined at $\sim 20: 1$ for the dual targeting step in EL250 cells propagating RP23-47M2 in order to favor a high likelihood of isolating clones with successful inactivation of $S f x n 1$. Initial attempts did not yield correct double recombinant clones, but instead, only the ChETA cassette with selection marker were found to be inserted in recovered BAC clones. The results were markedly improved by using high quality MAXI prep DNA of high concentration, ensuring the linearization was complete with no uncut plasmid remaining, and use of LB Lennox media instead of LB Miller in the recombineering protocol. With these changes the majority of experiments yielded one or more correct double recombinant BAC clones with insertion of the ChETA expression cassette downstream of the Drdla promoter region and inactivation of $S f x n 1$ by insertion of the three-frame stop mutation immediately downstream of the $S f x n 1$ start codon. The insertion of the inactivation cassette incorporated a new diagnostic SalI restriction site adjacent to the three-frame stop mutation, and thus, it was feasible to verify $S f x n 1$ inactivation at the DNA level by SalI digestion and PFGE (Figure 4F).

\section{PRESERVED CELL-TYPE SPECIFIC EXPRESSION PATTERNS IN BAC TRANSGENIC MICE CREATED WITH ADVANCED RECOMBINEERING STRATEGIES}

An important consideration for the BAC trimming and gene inactivation strategies employed here is whether or not the BAC DNA manipulations ultimately negatively impact the transgene expression pattern in transgenic animals. Although these strategies appear highly effective for removal of unwanted genes from
BAC clones, the utility of such manipulations would be greatly diminished if the cell type-specific expression patterns were not preserved. To demonstrate faithful expression we created BAC transgenic mouse lines for expression of the fast kinetic ChR2 variant ChETA under the regulatory elements for Adora2A (A2A), Drd1a (D1), and Slc6a3 (DAT). The BAC clones were modified as described using BAC trimming or gene inactivation strategies such that no unwanted extra genes were present. The modified BAC clones were used for pronuclear injection to create BAC transgenic founders. In each case we were able to successfully identify a single founder line for A2A-ChETA, D1-ChETA, and DAT-ChETA with functional transgene expression matching to the respective endogenous expression patterns (Figure 5). In order to further validate transgene expression in D1-ChETA mice we crossed these mice to the well characterized D1-EGFP or D2-EGFP GENSAT BAC transgenic mice. Our analysis revealed a near perfect overlap of EGFP and tdTomato (proxy for ChETA) restricted to striatonigral medium spiny neurons (MSNs) in D1EGFP/D1-ChETA double transgenic mice (Figures 6A,B). In contrast, virtually no overlap in EGFP and tdTomato expression was observed in the dorsal striatum region of D2-EGFP/D1-ChETA double transgenic mice indicating exclusion of ChETA expression from striatopallidal MSNs (Figures 6C,D).

\section{DISCUSSION}

We have designed and implemented BAC recombineering strategies for eliminating undesirable extra genes from BAC clones in order to circumvent confounds due to overexpression of extra genes in BAC transgenic lines. The BAC recombineering strategies are simple, efficient, and reproducible and have been implemented to successfully modify numerous mouse BAC clones from the RP23/RP24 library. Our BAC trimming strategy enables precisely targeted deletions of undesirable BAC DNA regions, such as those stretches harboring off-target extra genes. By judicious selection of BAC clones, in most cases it is feasible to remove all unwanted extra genes with a single deletion step targeting only one end (rather than both ends) of the BAC. We have demonstrated precise deletions of up to $111 \mathrm{~kb}$ and spanning as many as four unique genes in a single recombineering event with no evidence for any upper size limit thus far. Notably, excessive removal of large expanses of BAC DNA can be detrimental to the intended transgene expression pattern. Thus, although BAC trimming may be optimal for a wide variety of applications, it may not be feasible to fully remove all unwanted portions of BAC DNA for every BAC clone of interest.

In some BAC clones a gene of interest may occasionally be found to reside in very close proximity to a separate off-target gene, as was the case for the Drdla and Chat spanning BAC clones in our study. In such cases a simple BAC trimming strategy is not suitable since deletion of the unwanted extra gene would also eliminate putative $5^{\prime}$ or $3^{\prime}$ regulatory elements essential for specifying the unique expression pattern of the targeted gene. Thus, a gene inactivation strategy was implemented to insert a three-frame translational stop mutation plus diagnostic restriction site immediately downstream of the initiating methionine start codon in the unwanted extra gene. The size of the inactivation cassette is intentionally minimal (e.g., lacking antibiotic 

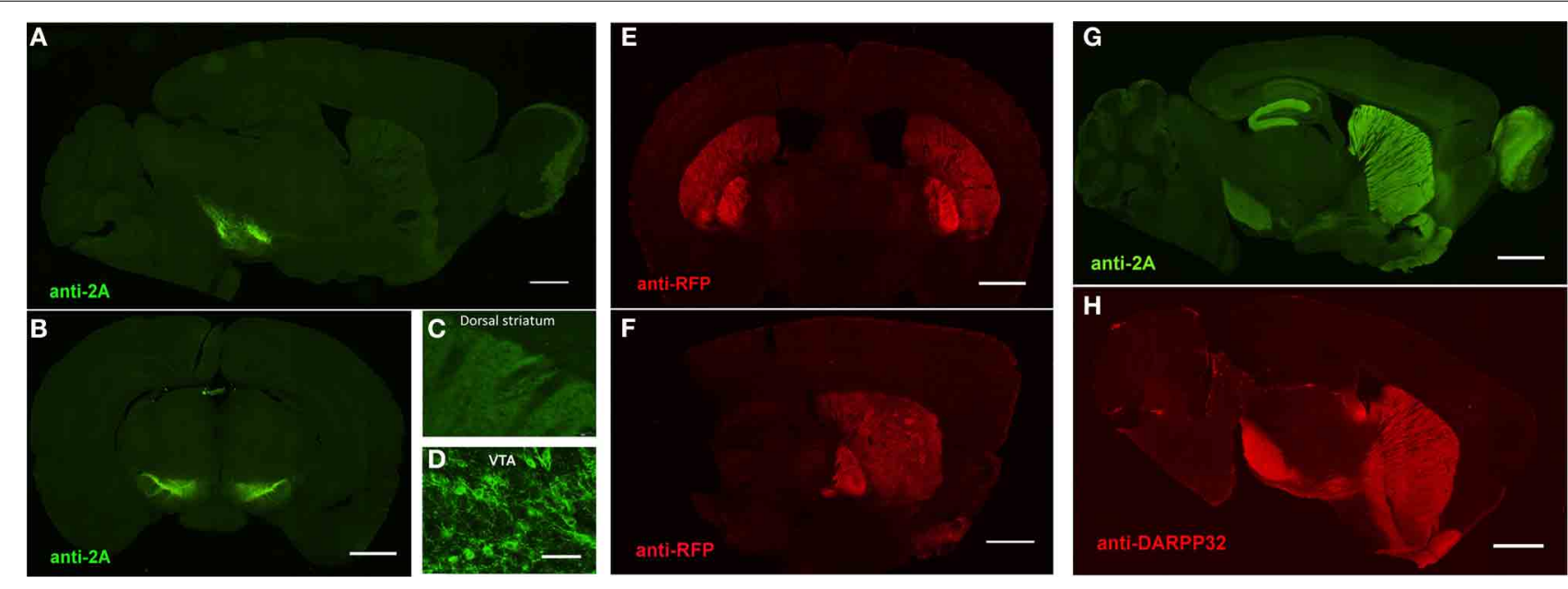

FIGURE 5 | Preserved cell-type specific expression patterns in BAC transgenic mice created with advanced recombineering strategies. (A-D) Anti-2A slice staining in brain slices from DAT-ChETA line 3 mice reveals the distribution of membrane-targeted ChETA protein. Sagittal $(\mathbf{A})$ and coronal (B) sections showing ChR2 expression in midbrain dopamine neurons. (C,D) High magnification images of axon terminal labeling in the dorsal striatum (C) and labeled neurons in the ventral tegmental area (D). (E,F) Anti-RFP slice staining in brain slices from A2A-ChETA line 13 mice showing tdTomato expression in striatopallidal medium spiny neurons (MSNs) in coronal (E) and sagittal (F) sections. Lighter expression can also be detected in putative cortical astrocytes. (G) Anti-2A slice staining in a sagittal brain slice from D1-ChETA line 1 mice showing ChETA expression in striatonigral MSNs. Additional expression is apparent in other brain regions, particularly the dentate gyrus, layer VI cortex, and olfactory bulb.

(F) Anti-DARPP32 slice staining to label both striatonigral and striatopallidal MSNs. Scale bars: $1 \mathrm{~mm}$ in (A,B,E-H) and $100 \mu \mathrm{m}$ in (C,D). selection cassette) and serves to eliminate functional gene expression from the unwanted extra gene without additional disruption to important regulatory elements in the region. The BAC recombineering strategy for gene inactivation requires dual targeting together with a second targeting cassette incorporating a functional selection marker. This dual targeting strategy allows for simultaneous insertion of a transgene cassette and inactivation of an extra gene by selecting for clones that have undergone successful homologous recombination at both locations within the BAC DNA sequence-i.e., double recombinant clones. In order to ensure a high likelihood that the antibiotic selection appropriately isolates double recombinant clones, the concentrations of each linearized targeting vector must be carefully proportioned. With optimization, the dual homologous recombination step was efficient enough to routinely identify multiple successfully targeted clones in a single attempt.

The overall goal of our efforts was to remove all extra genes from the selected BAC clones using either or both of these versatile recombineering strategies, leaving only a single targeted gene with sufficient $5^{\prime}$ and $3^{\prime}$ flanking regions to direct faithful gene expression in vivo in transgenic mice derived from pronuclear injection of the modified BAC DNA. In essence, the resultant modified BACs could be considered as designer "mini BACs" that retain only the most essential elements from the parent BAC clone while removing all non-essential portions. In designing our approaches we have had excellent initial success following a general guideline of retaining at least $50 \mathrm{~kb}$ of $5^{\prime}$ sequence and $15-20 \mathrm{~kb}$ of $3^{\prime}$ sequence flanking the targeted gene within BAC clones; however, the extent of $5^{\prime}$ and $3^{\prime}$ regions required must be determined empirically on a case-by-case basis. Once a successfully modified BAC clone is validated for proper cell-type specific expression, the same BAC modification strategy or preferably the same trimmed BAC clone itself can then be re-purposed for subsequent recombineering and expression of virtually any other transgene of interest. We provide a list of several BAC clones that have been modified using these methods and that can be utilized to target transgene expression to defined neuronal subsets (Table 2).

The approaches we have described for modifying BAC DNA using recombineering in E. coli build upon and extend the work of others. In three earlier studies BAC trimming (sometimes also called BAC shaving) was similarly accomplished by targeted replacement of a DNA region with a selection marker using homologous recombination (Hill et al., 2000; Testa et al., 2003, 2004). The end goal of such BAC DNA manipulation strategies was either to create precise vectors for traditional gene targeting in mouse embryonic stem cells or to reduce redundant effort in genome sequencing projects. In contrast, we applied BAC trimming to eliminate extra off-target genes so as to avoid potentially confounding effects in mice derived from pronuclear BAC DNA injections.

A substantially different method of retrieving mini BAC fragments was previously described. This method utilized PCR and gap repair in $E$. coli to subclone $\leq 80 \mathrm{~kb}$ BAC fragments into the low copy number pBR322 vector (Lee et al., 2001). However, the resulting transgene expression patterns were highly variable and did not fully recapitulate the endogenous gene expression patterns. This stands in contrast to our results demonstrating excellent preservation of the intended cell-type specific transgene expression patterns in transgenic mice derived from BAC DNA modified using our improved strategies. This difference is likely due to the strict size limitation for sub-cloning DNA fragments 


\section{D1-EGFP/D1-ChETA}
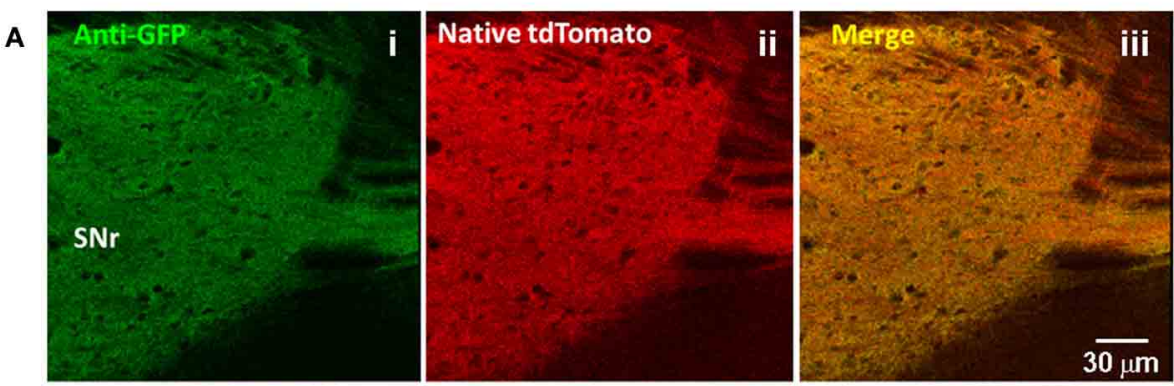

B
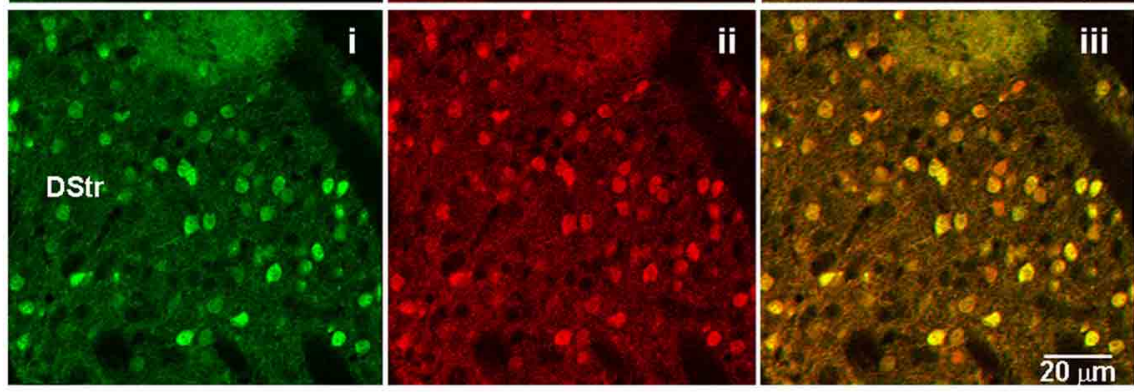

\section{D2-EGFP/D1-ChETA}
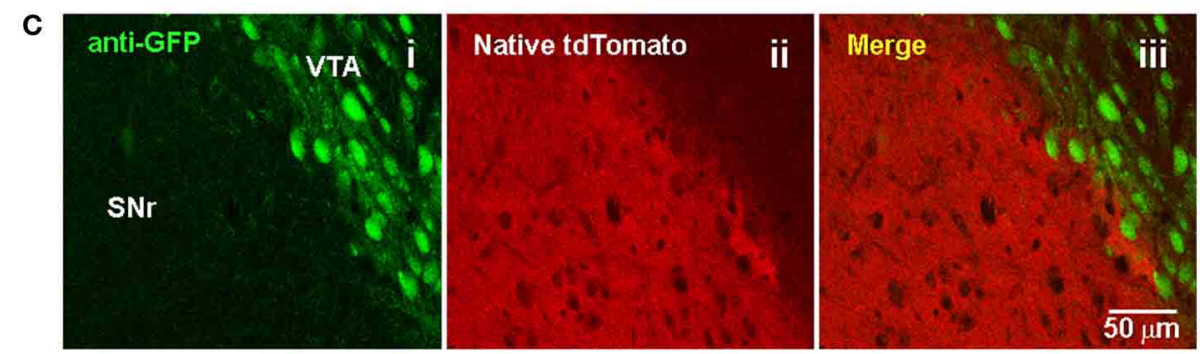

D
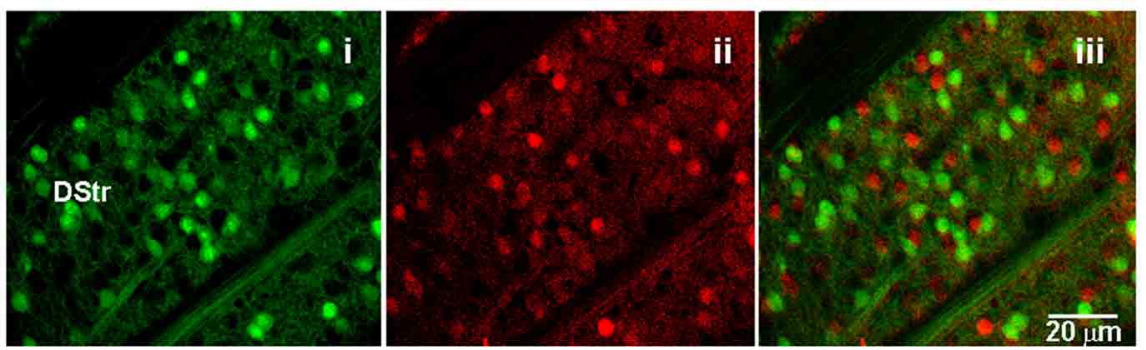

FIGURE 6 | Validation of transgene expression in striatonigral but not striatopallidal MSNs in D1-ChETA line 1 mice. (A,B) Confocal images in brain slices from D1-EGFP/Drd1a-ChETA double transgenic mice. (A) Substantia nigra pars reticulata ( $\mathrm{SNr}$ ) region showing anti-EGFP (Ai), native tdTomato fluorescence (Aii), and the merged image (Aiii). (B) Images from the dorsal striatum (DStr) region. (C,D) Confocal images in brain slices from D2-EGFP/D1-ChETA double transgenic mice. (C) SNrNTA boundary showing anti-EGFP (Ci), native tdTomato fluorescence (Cii), and the merged image (Ciii). (D) Images from the DStr region. into pBR322, whereas no practical size limitation is expected with typical BAC vectors such as pBACe3.6 and pTARBAC1, particularly since BAC DNA is being trimmed rather than retrieved by gap repair. Thus, the approaches that we have described seem to be more flexible when selection of considerably larger $5^{\prime}$ or $3^{\prime}$ regions surrounding the target expression cassette are required for achieving faithful expression in vivo, as is most often the case.
There are now extensive repositories of BAC transgenic mouse lines available, including over a thousand distinct Cre driver and EGFP reporter lines produced by the GENSAT project alone. We analyzed the full collection of BAC clones used for creating the extensive GENSAT Cre driver repository as a representative data set and determined that $75 \%$ of the BAC clones contain at least one or more extra genes. Even more surprisingly, one-third of the 
Table 2 | Successfully modified BAC clones using BAC trimming and gene inactivation approaches.

\begin{tabular}{|c|c|c|c|c|c|c|c|}
\hline $\begin{array}{l}\text { Targeted } \\
\text { gene }\end{array}$ & BAC ID & $\begin{array}{c}\text { BAC size } \\
\text { (kb) }\end{array}$ & $\begin{array}{l}\text { deletion } \\
\text { size (kb) }\end{array}$ & $\begin{array}{c}\text { trimmed } \\
\text { BAC size (kb) }\end{array}$ & deleted genes & inactivated genes & insertion size (bp) \\
\hline Drd1a & RP23-47M2 & 283.0 & - & - & - & Sfxn1^ & $29 \mathrm{bp}$ \\
\hline Adora2a & RP24-238K3 & 186.0 & 50.8 & 135.2 & 1110038D17Rik-210*, Upb1 & - & - \\
\hline Slc6a3 (DAT) & RP24-269I17 & 199.7 & 84.0 & 115.7 & Clptm1I, Tert, Slc6a18, Slc6a19* & - & - \\
\hline Chat & RP24-256F2 & 176.2 & 43.5 & 132.7 & 1700024G13Rik, Ercc6* & S/c18a3^ & $29 \mathrm{bp}$ \\
\hline
\end{tabular}

*Partial gene sequence only.

` Gene inactivation by insertion of a 3-frame triple STOP cassette.

BAC clones contained at least three or more extra genes and $5 \%$ contained at least nine or more extra genes. The most extreme case was a BAC clone that spanned fourteen different fully intact extra genes, and this clone was used to create both Cre driver and EGFP reporter lines (Thbs3-spanning BAC clone RP23-368D24). Despite the extensive proven utility of numerous GENSAT lines, it is an unfortunate possibility that many available BAC transgenic lines may suffer from confounds of varying degrees due in part to overexpression of one or more "extra" genes. In some cases the unintended overexpression of one or more extra genes in various tissues of transgenic animals may have profound consequences and implications for interpreting experimental results. In other cases the extra genes may not be expressed at functionally relevant levels or perhaps there may be no measurable impact despite high level expression (Heiman et al., 2008). In support of the former, recent experimental evidence has emerged claiming significant behavioral and functional alterations in several commonly used BAC transgenic mouse lines (Ade et al., 2011; Bagetta et al., 2011; Kramer et al., 2011; Kolisnyk et al., 2013), thereby raising concerns about some aspects of the findings from the numerous studies that have implemented these lines to date. Although some aspects of the reported defects have been disputed or reconciled (Chan et al., 2012; Nelson et al., 2012), this controversy highlights the need for more rigorous systematic characterization of BAC transgenic lines. In the study by Kolisnyk et al. (2013) it was clearly demonstrated that the measured behavioral and physiological alterations were the direct result of high BAC copy number insertion and unintended overexpression of VAChT in Chat-ChR2(H134R)-EYFP line 6 BAC transgenic animals (Kolisnyk et al., 2013). Given this finding, further detailed investigations are likely to reveal significant impacts of overexpressed extra genes in many other widely used BAC transgenic lines. This point underscores the potential benefits of the improved BAC recombineering strategies we have developed for removing or inactivating extra genes for the purpose of creating transgenic tools with fewer potential confounds.

Beyond the limited examples we have cited above, there are undoubtedly many commonly used BAC transgenic lines for which behavioral or physiological confounds are likely but have yet to be directly assessed. As an example, several BAC transgenic lines have been reported for transgene expression in cholinergic neurons using identical or highly similar Chat-spanning BAC clones as the one used in developing the Chat-ChR2(H134R)EYFP line 6 mice. These include a Chat-Cre driver line (Gong et al., 2007), two distinct Chat-EGFP reporter lines (Gong et al., 2003; Tallini et al., 2006), and a Chat-L10a-EGFP BacTRAP line (Doyle et al., 2008). Furthermore, a Chat-Cre BAC transgenic rat was developed by pronuclear injection of the identical Chat-Cre mouse BAC clone used to create the Chat-Cre mouse line (Witten et al., 2011). Thus, although no direct assessment of VAChT overexpression has been reported for these five independent lines, it is expected that all will have some degree of overexpression of VAChT (and potentially Ogdhl and 1700024G13Rik) depending on the BAC transgene copy number for each line. However, it is worth noting that high BAC copy number insertions as reported for the ChAT-ChR2(H134R)-EYFP line 6 tend to be far less common as opposed to low BAC copy number insertions.

In the long-term, we propose that the most effective strategy will be to re-engineer and develop new BAC transgenic lines using improved BAC recombineering strategies to eliminate nonessential portions of BAC DNA, including (but not limited to), methods described in this study, and to deposit validated mini BACs into an open source repository for widespread use. The versatile new resources we have developed (e.g., BAC modification vectors and successfully modified BAC clones) and the simple and efficient methods we have demonstrated should facilitate progress in this area by providing further options to support diverse recombineering needs. Furthermore, with advances in pronuclear injection-based targeted transgenesis (Ohtsuka et al., 2010; Tasic et al., 2011) and identification of "safe" genomic docking loci permissible for high level expression, it may soon be possible to develop BAC transgenic animals that are free of both confounding extra genes and detrimental positional effects from random transgene integration. Continued technological innovation will help to ensure an ever-expanding collection of highly useful BAC transgenic tools that can pave the way to deciphering the functions of the remarkably diverse cell types in the brain.

\section{ACKNOWLEDGMENTS}

We wish to acknowledge Dr. Bernd Gloss for providing the iTV1 plasmid used for constructing BAC targeting vectors and for discussions on the BAC recombineering procedures described in this work. We thank Dr. Karl Deisseroth for providing the original ChR2(E123T/H134R)-EYFP DNA construct. The EL250 strain 
used for BAC recombineering was generously provided by Dr. Neal Copeland. We thank Peimin Qi and the members of the MIT Transgenic Facility for excellent technical support related to pronuclear injection of BAC DNA. We thank Jill R. Crittenden for critical comments on this manuscript. This work was supported in part by a National Alliance for Research on Schizophrenia and Depression: The Brain and Behavior Research Foundation Young Investigator Award to Jonathan T. Ting, and U.S. National Institutes of Health Ruth L. Kirschstein National Research Service Awards to Jonathan T. Ting (F32-MH084460).

\section{REFERENCES}

Ade, K. K., Wan, Y., Chen, M., Gloss, B., and Calakos, N. (2011). An improved BAC transgenic fluorescent reporter line for sensitive and specific identification of striatonigral medium spiny neurons. Front. Syst. Neurosci. 5:32. doi: $10.3389 /$ fnsys.2011.00032

Aponte, Y., Atasoy, D., and Sternson, S. M. (2011). AGRP neurons are sufficient to orchestrate feeding behavior rapidly and without training. Nat. Neurosci. 14, 351-355. doi: 10.1038/nn.2739

Atasoy, D., Aponte, Y., Su, H. H., and Sternson, S. M. (2008). A FLEX switch targets Channelrhodopsin-2 to multiple cell types for imaging and long-range circuit mapping. J. Neurosci. 28, 7025-7030. doi: 10.1523/JNEUROSCI.1954-08.2008

Bagetta, V., Picconi, B., Marinucci, S., Sgobio, C., Pendolino, V., Ghiglieri, V., et al. (2011). Dopamine-dependent long-term depression is expressed in striatal spiny neurons of both direct and indirect pathways: implications for Parkinson's disease. J. Neurosci. 31, 12513-12522. doi: 10.1523/JNEUROSCI.2236-11.2011

Belforte, J. E., Zsiros, V., Sklar, E. R., Jiang, Z., Yu, G., Li, Y., et al. (2010). Postnatal NMDA receptor ablation in corticolimbic interneurons confers schizophrenialike phenotypes. Nat. Neurosci. 13, 76-83. doi: 10.1038/nn.2447

Bian, Q., and Belmont, A. S. (2010). BAC TG-EMBED: one-step method for high-level, copy-number-dependent, position-independent transgene expression. Nucleic Acids Res. 38, e127. doi: 10.1093/nar/gkq178

Bock, R., Shin, J. H., Kaplan, A. R., Dobi, A., Markey, E., Kramer, P. F., et al. (2013). Strengthening the accumbal indirect pathway promotes resilience to compulsive cocaine use. Nat. Neurosci. 16, 632-638. doi: 10.1038/nn.3369

Cardin, J. A., Carlen, M., Meletis, K., Knoblich, U., Zhang, F., Deisseroth, K., et al. (2009). Driving fast-spiking cells induces gamma rhythm and controls sensory responses. Nature 459, 663-667. doi: 10.1038/nature08002

Chan, C. S., Peterson, J. D., Gertler, T. S., Glajch, K. E., Quintana, R. E., Cui, Q., et al. (2012). Strain-specific regulation of striatal phenotype in Drd2-eGFP BAC transgenic mice. J. Neurosci. 32, 9124-9132. doi: 10.1523/JNEUROSCI.022912.2012

Chao, H. T., Chen, H., Samaco, R. C., Xue, M., Chahrour, M., Yoo, J., et al. (2010). Dysfunction in GABA signalling mediates autism-like stereotypies and Rett syndrome phenotypes. Nature 468, 263-269. doi: 10.1038/nature09582

Chaudhury, D., Walsh, J. J., Friedman, A. K., Juarez, B., Ku, S. M., Koo, J. W., et al. (2013). Rapid regulation of depression-related behaviours by control of midbrain dopamine neurons. Nature 493, 532-536. doi: 10.1038/nature11713

Cui, G., Jun, S. B., Jin, X., Pham, M. D., Vogel, S. S., Lovinger, D. M., et al. (2013). Concurrent activation of striatal direct and indirect pathways during action initiation. Nature 494, 238-242. doi: 10.1038/nature1 1846

Doyle, J. P., Dougherty, J. D., Heiman, M., Schmidt, E. F., Stevens, T. R., Ma, G., et al. (2008). Application of a translational profiling approach for the comparative analysis of CNS cell types. Cell 135, 749-762. doi: 10.1016/j.cell.2008.10.029

Farrell, M. S., Pei, Y., Wan, Y., Yadav, P. N., Daigle, T. L., Urban, D. J., et al. (2013). A Galphas DREADD mouse for selective modulation of cAMP production in striatopallidal neurons. Neuropsychopharmacology 38, 854-862. doi: 10.1038/npp.2012.251

Gong, S., Doughty, M., Harbaugh, C. R., Cummins, A., Hatten, M. E., Heintz, N., et al. (2007). Targeting Cre recombinase to specific neuron populations with bacterial artificial chromosome constructs. J. Neurosci. 27, 9817-9823. doi: 10.1523/JNEUROSCI.2707-07.2007

Gong, S., Zheng, C., Doughty, M. L., Losos, K., Didkovsky, N., Schambra, U. B., et al. (2003). A gene expression atlas of the central nervous system based on bacterial artificial chromosomes. Nature 425, 917-925. doi: 10.1038/nature02033

Hagglund, M., Borgius, L., Dougherty, K. J., and Kiehn, O. (2010). Activation of groups of excitatory neurons in the mammalian spinal cord or hindbrain evokes locomotion. Nat. Neurosci. 13, 246-252. doi: 10.1038/nn.2482
Hagglund, M., Dougherty, K. J., Borgius, L., Itohara, S., Iwasato, T., and Kiehn, O. (2013). Optogenetic dissection reveals multiple rhythmogenic modules underlying locomotion. Proc. Natl. Acad. Sci. U.S.A. 110, 11589-11594. doi: 10.1073/pnas.1304365110

Halassa, M. M., Siegle, J. H., Ritt, J. T., Ting, J. T., Feng, G., and Moore, C. I. (2011). Selective optical drive of thalamic reticular nucleus generates thalamic bursts and cortical spindles. Nat. Neurosci. 14, 1118-1120. doi: 10.1038/nn.2880

Heiman, M., Schaefer, A., Gong, S., Peterson, J. D., Day, M., Ramsey, K. E., et al. (2008). A translational profiling approach for the molecular characterization of CNS cell types. Cell 135, 738-748. doi: 10.1016/j.cell.2008.10.028

Heintz, N. (2004). Gene expression nervous system atlas (GENSAT). Nat. Neurosci. 7, 483. doi: $10.1038 / \mathrm{nn} 0504-483$

Hill, F., Benes, V., Thomasova, D., Stewart, A. F., Kafatos, F. C., and Ansorge, W. (2000). BAC trimming: minimizing clone overlaps. Genomics 64, 111-113. doi: 10.1006/geno.1999.6106

Kim, J. H., Lee, S. R., Li, L. H., Park, H. J., Park, J. H., Lee, K. Y., et al. (2011). High cleavage efficiency of a $2 \mathrm{~A}$ peptide derived from porcine teschovirus-1 in human cell lines, zebrafish and mice. PLoS ONE 6:e18556. doi: 10.1371/journal.pone.0018556

Kolisnyk, B., Guzman, M. S., Raulic, S., Fan, J., Magalhaes, A. C., Feng, G., et al. (2013). ChAT-ChR2-EYFP mice have enhanced motor endurance but show deficits in attention and several additional cognitive domains. J. Neurosci. 33, 10427-10438. doi: 10.1523/JNEUROSCI.0395-13.2013

Kramer, P. F., Christensen, C. H., Hazelwood, L. A., Dobi, A., Bock, R., Sibley, D. R., et al. (2011). Dopamine D2 receptor overexpression alters behavior and physiology in Drd2-EGFP mice. J. Neurosci. 31, 126-132. doi: 10.1523/JNEUROSCI.4287-10.2011

Kravitz, A. V., Freeze, B. S., Parker, P. R., Kay, K., Thwin, M. T., Deisseroth, K., et al. (2010). Regulation of parkinsonian motor behaviours by optogenetic control of basal ganglia circuitry. Nature 466, 622-626. doi: 10.1038/nature09159

Lee, E. C., Yu, D., Martinez De Velasco, J., Tessarollo, L., Swing, D. A., Court, D. L., et al. (2001). A highly efficient Escherichia coli-based chromosome engineering system adapted for recombinogenic targeting and subcloning of BAC DNA. Genomics 73, 56-65. doi: 10.1006/geno.2000.6451

Lenz, J. D., and Lobo, M. K. (2013). Optogenetic insights into striatal function and behavior. Behav. Brain Res. 255, 44-54. doi: 10.1016/j.bbr.2013.04.018

Lobo, M. K., Karsten, S. L., Gray, M., Geschwind, D. H., and Yang, X. W. (2006). FACS-array profiling of striatal projection neuron subtypes in juvenile and adult mouse brains. Nat Neurosci. 9, 443-452. doi: 10.1038/nn1654

Ma, M., and Luo, M. (2012). Optogenetic activation of Basal forebrain cholinergic neurons modulates neuronal excitability and sensory responses in the main olfactory bulb. J. Neurosci. 32, 10105-10116. doi: 10.1523/JNEUROSCI.005812.2012

Madisen, L., Mao, T., Koch, H., Zhuo, J. M., Berenyi, A., Fujisawa, S., et al. (2012). A toolbox of Cre-dependent optogenetic transgenic mice for light-induced activation and silencing. Nat. Neurosci. 15, 793-802. doi: 10.1038/nn.3078

Nelson, A. B., Hang, G. B., Grueter, B. A., Pascoli, V., Luscher, C., Malenka, R. C., et al. (2012). A comparison of striatal-dependent behaviors in wild-type and hemizygous Drdla and Drd2 BAC transgenic mice. J. Neurosci. 32, 9119-9123. doi: 10.1523/JNEUROSCI.0224-12.2012

Nieh, E. H., Kim, S. Y., Namburi, P., and Tye, K. M. (2013). Optogenetic dissection of neural circuits underlying emotional valence and motivated behaviors. Brain Res. 1511, 73-92. doi: 10.1016/j.brainres.2012.11.001

Ohtsuka, M., Ogiwara, S., Miura, H., Mizutani, A., Warita, T., Sato, M., et al. (2010). Pronuclear injection-based mouse targeted transgenesis for reproducible and highly efficient transgene expression. Nucleic Acids Res. 38, e198. doi: 10.1093/nar/gkq860

Osoegawa, K., Tateno, M., Woon, P. Y., Frengen, E., Mammoser, A. G., Catanese, J. J., et al. (2000). Bacterial artificial chromosome libraries for mouse sequencing and functional analysis. Genome Res. 10, 116-128. doi: 10.1101/gr.10.1.116

Petreanu, L., Mao, T., Sternson, S. M., and Svoboda, K. (2009). The subcellular organization of neocortical excitatory connections. Nature 457, 1142-1145. doi: 10.1038/nature07709

Ren, J., Qin, C., Hu, F., Tan, J., Qiu, L., Zhao, S., et al. (2011). Habenula cholinergic neurons co-release glutamate and acetylcholine and activate postsynaptic neurons via distinct transmission modes. Neuron 69, 445-452. doi: 10.1016/j.neuron.2010.12.038

Saunders, A., Johnson, C. A., and Sabatini, B. L. (2012). Novel recombinant adenoassociated viruses for Cre activated and inactivated transgene expression in neurons. Front. Neural Circuits 6:47. doi: 10.3389/fncir.2012.00047 
Stamatakis, A. M., Jennings, J. H., Ung, R. L., Blair, G. A., Weinberg, R. J., Neve, R. L., et al. (2013). A unique population of ventral tegmental area neurons inhibits the lateral habenula to promote reward. Neuron 80, 1039-1053. doi: 10.1016/j.neuron.2013.08.023

Steinberg, E. E., Keiflin, R., Boivin, J. R., Witten, I. B., Deisseroth, K., and Janak, P. H. (2013). A causal link between prediction errors, dopamine neurons and learning. Nat. Neurosci. 16, 966-973. doi: 10.1038/nn.3413

Tai, L. H., Lee, A. M., Benavidez, N., Bonci, A., and Wilbrecht, L. (2012). Transient stimulation of distinct subpopulations of striatal neurons mimics changes in action value. Nat. Neurosci. 15, 1281-1289. doi: 10.1038/nn.3188

Tallini, Y. N., Shui, B., Greene, K. S., Deng, K. Y., Doran, R., Fisher, P. J., et al. (2006). BAC transgenic mice express enhanced green fluorescent protein in central and peripheral cholinergic neurons. Physiol. Genomics 27, 391-397. doi: 10.1152/physiolgenomics.00092.2006

Tan, K. R., Yvon, C., Turiault, M., Mirzabekov, J. J., Doehner, J., Labouebe, G., et al. (2012). GABA neurons of the VTA drive conditioned place aversion. Neuron 73, 1173-1183. doi: 10.1016/j.neuron.2012.02.015

Tasic, B., Hippenmeyer, S., Wang, C., Gamboa, M., Zong, H., Chen-Tsai, Y., et al. (2011). Site-specific integrase-mediated transgenesis in mice via pronuclear injection. Proc. Natl. Acad. Sci. U.S.A. 108, 7902-7907. doi: 10.1073/pnas.10195 07108

Testa, G., Vintersten, K., Zhang, Y., Benes, V., Muyrers, J. P., and Stewart, A. F. (2004). BAC engineering for the generation of ES cell-targeting constructs and mouse transgenes. Methods Mol. Biol. 256, 123-139. doi: 10.1385/1-59259-753$\mathrm{X}: 123$

Testa, G., Zhang, Y., Vintersten, K., Benes, V., Pijnappel, W. W., Chambers, I., et al. (2003). Engineering the mouse genome with bacterial artificial chromosomes to create multipurpose alleles. Nat. Biotechnol. 21, 443-447. doi: 10.1038/nbt804

Tsai, H. C., Zhang, F., Adamantidis, A., Stuber, G. D., Bonci, A., De Lecea, L., et al. (2009). Phasic firing in dopaminergic neurons is sufficient for behavioral conditioning. Science 324, 1080-1084. doi: 10.1126/science.1168878

Tye, K. M., and Deisseroth, K. (2012). Optogenetic investigation of neural circuits underlying brain disease in animal models. Nat. Rev. Neurosci. 13, 251-266. doi: 10.1038/nrn3171

Witten, I. B., Lin, S. C., Brodsky, M., Prakash, R., Diester, I., Anikeeva, P., et al. (2010). Cholinergic interneurons control local circuit activity and cocaine conditioning. Science 330, 1677-1681. doi: 10.1126/science.1193771
Witten, I. B., Steinberg, E. E., Lee, S. Y., Davidson, T. J., Zalocusky, K. A., Brodsky, M., et al. (2011). Recombinase-driver rat lines: tools, techniques, and optogenetic application to dopamine-mediated reinforcement. Neuron 72 , 721-733. doi: 10.1016/j.neuron.2011.10.028

Yang, X. W., Model, P., and Heintz, N. (1997). Homologous recombination based modification in Escherichia coli and germline transmission in transgenic mice of a bacterial artificial chromosome. Nat. Biotechnol. 15, 859-865. doi: $10.1038 /$ nbt0997-859

Yizhar, O. (2012). Optogenetic insights into social behavior function. Biol. Psychiatry 71, 1075-1080. doi: 10.1016/j.biopsych.2011.12.029

Yizhar, O., Fenno, L. E., Prigge, M., Schneider, F., Davidson, T. J., O’Shea, D. J., et al. (2011). Neocortical excitation/inhibition balance in information processing and social dysfunction. Nature 477, 171-178. doi: 10.1038/nature10360

Zariwala, H. A., Borghuis, B. G., Hoogland, T. M., Madisen, L., Tian, L., De Zeeuw, C. I., et al. (2012). A Cre-dependent GCaMP3 reporter mouse for neuronal imaging in vivo. J. Neurosci. 32, 3131-3141. doi: 10.1523/JNEUROSCI.446911.2012

Zhao, S., Ting, J. T., Atallah, H. E., Qiu, L., Tan, J., Gloss, B., et al. (2011). Cell type-specific channelrhodopsin-2 transgenic mice for optogenetic dissection of neural circuitry function. Nat. Methods 8, 745-752. doi: 10.1038/ nmeth.1668

Conflict of Interest Statement: The authors declare that the research was conducted in the absence of any commercial or financial relationships that could be construed as a potential conflict of interest.

Received: 16 January 2014; accepted: 15 March 2014; published online: 03 April 2014. Citation: Ting JT and Feng G (2014) Recombineering strategies for developing next generation BAC transgenic tools for optogenetics and beyond. Front. Behav. Neurosci. 8:111. doi: 10.3389/fnbeh.2014.00111

This article was submitted to the journal Frontiers in Behavioral Neuroscience.

Copyright (C) 2014 Ting and Feng. This is an open-access article distributed under the terms of the Creative Commons Attribution License (CC BY). The use, distribution or reproduction in other forums is permitted, provided the original author(s) or licensor are credited and that the original publication in this journal is cited, in accordance with accepted academic practice. No use, distribution or reproduction is permitted which does not comply with these terms. 